\title{
ARTICLE
}

\section{Refinement of the RP17 locus for autosomal dominant retinitis pigmentosa, construction of a YAC contig and investigation of the candidate gene retinal fascin}

\author{
Soraya B ardien-K ruger ${ }^{1}$, Jacquie G reenberg ${ }^{1}$, B enjamin Tubb ${ }^{2}$, J oseph Bryan ${ }^{2}$, \\ Lurdes Q ueimado ${ }^{3}$, M ichael L ovett ${ }^{3}$ and R ajkumar S R amesar ${ }^{1}$ \\ ${ }^{1} D$ epartment of H uman G enetics, U niversity of Cape Town, South A frica \\ ${ }^{2} \mathrm{D}$ epartment of Cell Biology, B aylor College of M edicine, H ouston \\ ${ }^{3} \mathrm{M}$ cD ermott Center, U niversity of Texas Southwestern M edical Center, D allas, Texas, USA
}

\begin{abstract}
The R P17 locus for autosomal dominant retinitis pigmentosa has previously been mapped to chromosome 17q by linkage analysis. Two unrelated South A frican families are linked to this locus and the identification of key recombination events assigned the R P 17 locus to a $10 \mathrm{cM}$ interval on 17q22. The work reported here refines the mapping of the locus from a $10 \mathrm{cM}$ to a $1 \mathrm{cM}$ interval between the microsatellite markers D 17S1604 and D 17S948. A physical map of this interval was constructed using information from the W hitehead/M IT YAC contig WC 17.8. Sequence-tagged site (ST S) content mapping of seven overlapping Y AC s from this contig was employed in order to build the map. A BAC library was screened to cover a gap in the YAC contig and two positive BACs were identified. Intragenic polymorphisms in the retinal fascin gene provided evidence for the exclusion of this candidate as the R P17 disease gene.
\end{abstract}

Keywords: retinitis pigmentosa; R P 17; fine mapping; YAC contig; retinal fascin

\section{Introduction}

$R$ etinitis pigmentosa ( $R P$ ) is a generic name for a group of hereditary disorders that cause progressive retinal degeneration, usually leading to blindness in later life. Patients with RP characteristically experience night blindness, loss of peripheral vision and a decrease in contrast sensitivity. RP segregates as an autosomal dominant ( $\operatorname{adR} P)$, autosomal recessive or an $\mathrm{X}$-linked trait. The disorder may also occur in association with

Correspondence: A ssoc. Prof. R S R amesar, D epartment of $H$ uman G enetics, U niversity of Cape Town M edical School, A nzio R oad, O bservatory, 7925 Cape Town, South A frica. Tel: 27214066 506; Fax: 27214480 906; E -mail: rr@anat.uct.ac.za R eceived 10 A ugust 1998; revised 230 ctober 1998; accepted 3 November 1998 certain syndromic disorders, eg $U$ sher and R efsum syndromes. ${ }^{1} \mathrm{~N}$ ine distinct loci have been mapped for $\operatorname{adR} P$, including the rhodopsin and peripherin-RDS genes and anonymous loci designated R P 1, R P9, R P 10, RP11, R P13, R P17 and R P18. 2-4 $^{-2}$

The R P17 locus was first reported in 1995 in a South A frican family of German descent. ${ }^{3}$ A second South A frican adR P family was later shown to be linked to this locus. ${ }^{5}$ The two families share a common haplotype that is associated with the disease phenotype, thereby suggesting descent of the disease gene from a common ancestor. By combining the linkage data and using haplotype analysis of recombinant chromosomes in 17 newly recruited family members, the RP17 critical interval was reduced. 
In order to assemble a physical map of the RP17 critical interval, the Whitehead Institute/M IT Center for $\mathrm{G}$ enome R esearch (http://www.genome.wi.mit.edu/) physical map data of YAC contig WC 17.8 was used. ${ }^{6}$ Sequence-tagged site (STS) content mapping was performed using CEPH mega-YACS, and STSs and expressed sequence tags (ESTS) from the R P 17 region. The refined localisation of the R P17 locus, in conjunction with an overlapping Y A C-based contig coverage of the critical interval, should facilitate the identification of this disease gene.

In combination with positional cloning approaches, candidate gene screening has also been undertaken to identify the RP17 gene. The retinal fascin gene was examined as a candidate because it is expressed exclusively in photoreceptor cells ${ }^{7}$ and has been mapped to $17 q$ (Tubb et al, in preparation).

\section{Materials and Methods}

\section{Patient Material and DNA Analysis}

B lood was collected from 44 members for family R PD 8, and from 25 individuals from family RPD 19, and DNA was extracted for linkage analysis using standard techniques. $M$ icrosatellite markers were typed on all the D NA samples by PCR with random incorporation of $\left[\alpha^{32} P\right] d C T P$ during the reaction. The amplified products (alleles) were resolved by size fractionation on $6 \%$ polyacrylamide denaturing gels and visualised by autoradiography. The pedigree relationships in both families were confirmed by the appropriate segregation of all the tested microsatellite markers.

\section{YAC-based STS-content Mapping \\ Primers for 9ST Ss and 3E ST s were used to screen the CE PH $M$ ega YAC Library. The YACs were selected from the Whitehead/MIT YAC contig WC 17.8. Colony PCR was performed on YA C s grown on selective (UR A -, TR P-) media, and the PCR products were resolved on $2 \%$ agarose gels and detected with ethidium bromide staining.}

\section{BAC-based STS-content Mapping}

The Human Bacterial A rtificial Chromosome DNA Pools R elease III (R esearch Genetics, Inc., H untsville, A L, USA) were screened according to the manufacturer's instructions. PCR products were electrophoresed on $1 \%$ agarose gels.

\section{Vectorette End Fragment Isolation Method}

End probes were generated from BAC 100E 23 and BAC $229 \mathrm{~L} 19$ insert DNA using a modified vectorette-PCR procedure. ${ }^{8}$ The BAC DNA was digested with Rsal, Pvull and $\mathrm{E}$ coRV and then ligated with the blunt-end vectorette cassette as described. ${ }^{8} \mathrm{U}$ sing these three vectorette libraries as templates, PCR was performed with a universal vectorette primer (5' TCT CCC TTC TCg A AT CgT AAC CgT TCg TAC $3^{\prime}$ ) and either primer T 7 or SP 6 to amplify the $5^{\prime}$ or the $3^{\prime}$ arm, respectively. The resulting PCR products obtained adjacent to the T 7 vector end for BA C 100E 23 and adjacent to the SP 6 end for BA C 229L 19, respectively, were gel-eluted using a QIA quick Gel Extraction K it (Q iagen, Germany; purchased from: Southern Cross Biotechnology (Pty) Ltd., Cape Town, SA ) and labelled with $\left[\alpha^{32} P\right] d C T P$ before being used as a probe. One microlitre of each of the seven YACS and the two BA C s comprising the R P 17 contig was dotted on to a $\mathrm{Hybond}-\mathrm{N}^{+}$membrane (A mersham; purchased from AEC A mersham (Pty) Ltd., SA) and hybridised to the purified vectorette $P C R$ product overnight. The blot was washed to a final stringency of $0.6 \times \mathrm{SSC} ; 0.3 \% \mathrm{SD} \mathrm{S}$ at $65^{\circ} \mathrm{C}$ and exposed to $X$-ray film (A gfa Curix, G ermany; purchased from: A gfa Division of B ayer (Pty) Ltd., SA ) for $20 \mathrm{~h}$.

\section{SSCP Analysis and DNA Sequencing of Retinal \\ Fascin}

Two polymorphisms found in the 5 ' putative promoter region and an intragenic polymorphism found in exon 1 of the retinal fascin gene were genotyped in the two adR $P$ families using standard SSC $P$ techniques. ${ }^{9}$ The allele frequencies observed for the two $5^{\prime}$ polymorphisms are 0.84 and 0.16 for an A to $G$ transversion $707 \mathrm{bp}$ upstream of the initiation codon; and 0.96 and 0.04 for a $C$ to $G$ transition $622 \mathrm{bp}$ upstream of the initiation codon (Tubb et al, in preparation). These two polymorphisms were amplified and detected in a single PCR product using the following primers, forward-5' gAC gTC gA C A gg CTg CA C ggC CAC TgT gT 3' and reverse-5' TgA TCC CCT TTT CCT CAT A gC 3'. The polymorphism in exon 1 is a T to $C$ transition located $738 \mathrm{bp} 3^{\prime}$ of the initiation codon. The PCR primers used to amplify this polymorphism are: forward-5' gCA TgA ATT C gA gCC gAC ggT ACT gCC TCA A gT CC $3^{\prime}$ and reverse-5' CgT A gT CgA CCT TgC CgC ACA gA g ACg TA g Cgg Tg 3'.

Primers were designed to PCR amplify the 5 exons of retinal fascin and the products were sequenced on an $A B$ PRISM 310 G enetic A nalyser (Perkin E Imer, N orwalk, CT, USA ).

\section{Results}

A total of 44 members from family RPD 8 (Figure 1a) and 25 members from family R PD 19 (Figure $1 b$ ) were genotyped with microsatellite markers from 17q22. The microsatellite markers used in the study and the genetic distances between them are illustrated in Figure $2 .{ }^{10}$ The analysis of recombinant events in informative meioses allowed for the fine mapping of the R P17 locus from a $10 \mathrm{cM}$ to a $1 \mathrm{cM}$ critical interval. O ne individual, V-15 (Figure 1a) is recombinant for the markers D 17S790 through to D 17S1604. In this individual the maternally inherited chromosome had recombined. $\mathrm{He}$ is an unequivocally affected 55-year-old male and on dilated fundal examination, was found to have the typical features of retinitis pigmentosa (including waxy pallor of the optic nerve heads, retinal vascular attenuation and some retinal pigment epithelial atrophy). This recombinant provides evidence that the $\operatorname{adR} P$ disease gene maps distal to D 17S1604. 


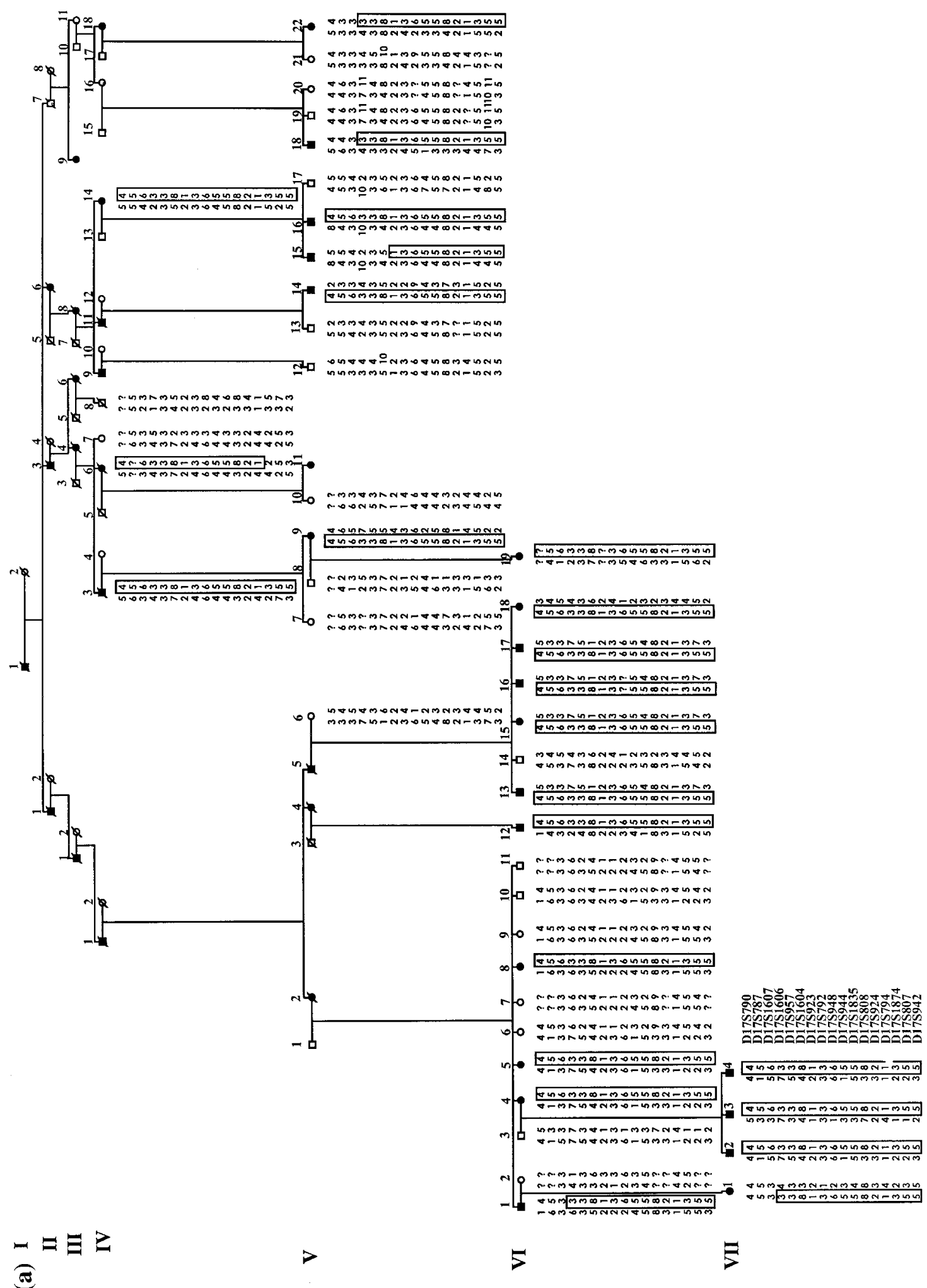

Figure 1 Pedigrees of the two kindreds, (a) family R PD 8 and (b) family R PD 19 linked to the RP 17 locus. The haplotype associated with the adR P disease phenotype is depicted in a box beneath each individual. In family R PD 19, individuals III -8, IV-5, IV-6, and IV-7 inherit the disease-associated haplotype for markers D 175948 to D $17 S 942$ but they do not exhibit any manifestations of RP (see text) 


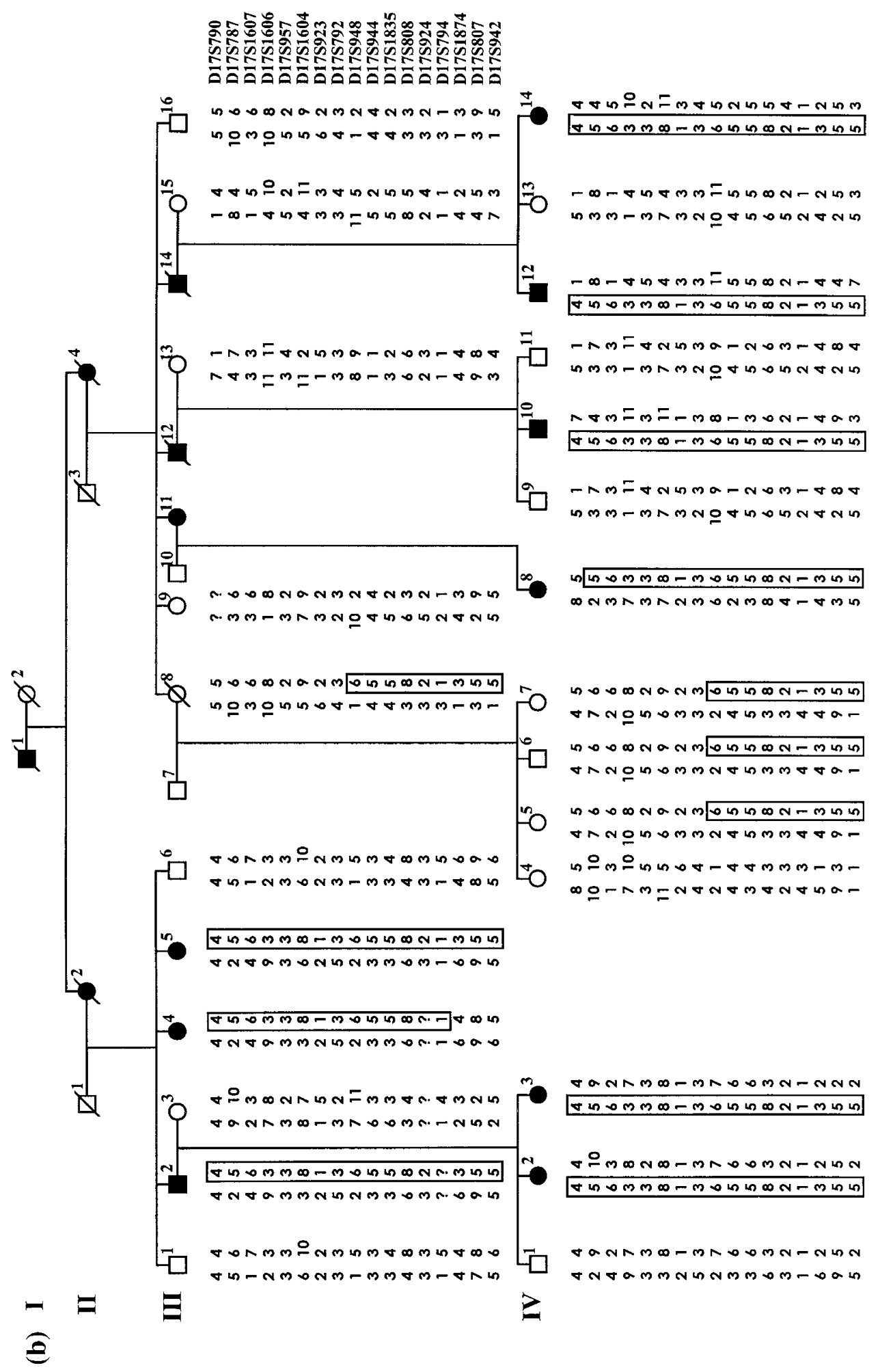

Figure 1 Continued

Similarly, individual III-8 (Figure 1 b) is recombinant for the markers D 175948 through to D 17S942. In this individual the maternally inherited chromosome had recombined. She died at age 73 , and had evidently, 


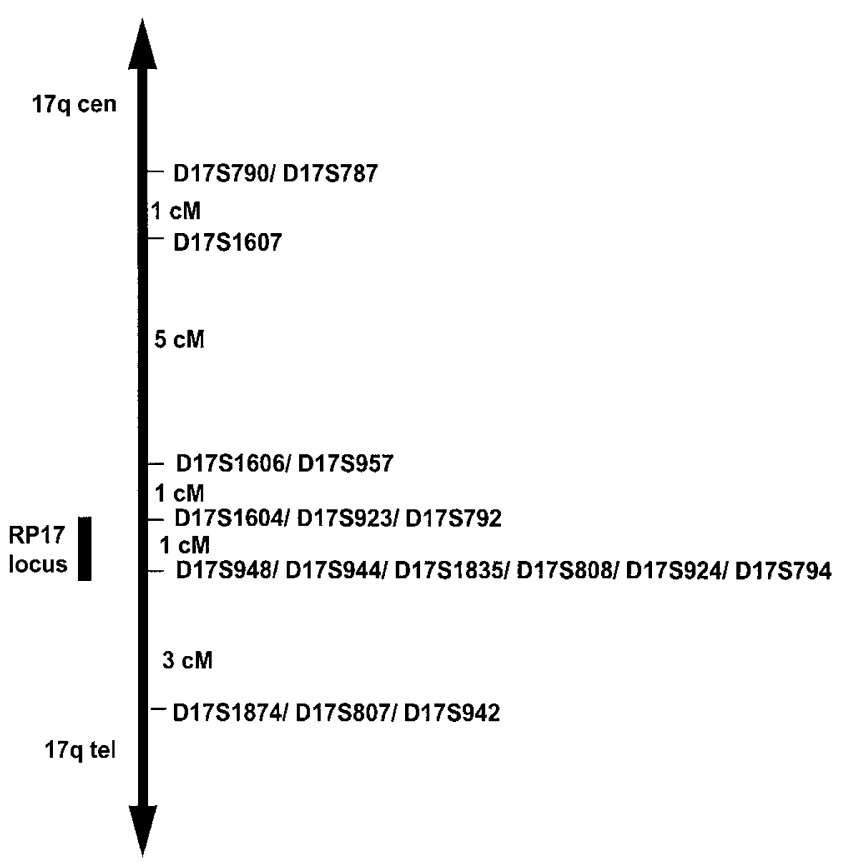

Figure 2 Schematic diagram depicting the positions of the microsatellite markers on 17q22 used in the study as well as the genetic distances calculated between adjacent loci. Markers separated by a slash (/) could be separated genetically using the $\mathrm{CEPH}$ reference families except for the presence of cataracts, not shown any signs of visual problems up to her death. The construction of haplotypes indicates that three of her four offspring (individuals IV -5, IV -6 and IV-7) had inherited the recombinant allele from their mother. They are all over 50 years of age (the mean age of onset of R P in the family is between 20 and 30 years of age) and were subsequently referred to an ophthalmologist for examination. No abnormality suggestive of retinitis pigmentosa was detected. The haplotype data presented here therefore provides evidence that the $\operatorname{adR} P$ disease gene maps proximal to D $17 S 948$.

By combining the haplotype data obtained from the two families, the minimal co-segregating region for R P17 was refined to a $1 \mathrm{cM}$ interval between markers D 17S1604 and D 17S948. A physical map of this $1 \mathrm{cM}$ interval was constructed using the Whitehead/M IT database and the CEPH Mega YAC library. STScontent analysis was employed to construct a contig of seven overlapping YACS as illustrated in Figure 3. A total of 9STSs and 3ESTs from the RP17 critical interval were used and the YA C s were ordered according to their marker content. $U$ pon further investigation, it was revealed that there was a gap in the YAC contig between markers WI-6805 and CHLC.GATA $11 \mathrm{C} 11$ and an attempt was made to cover this interval by screening a BAC library. Two positive clones were

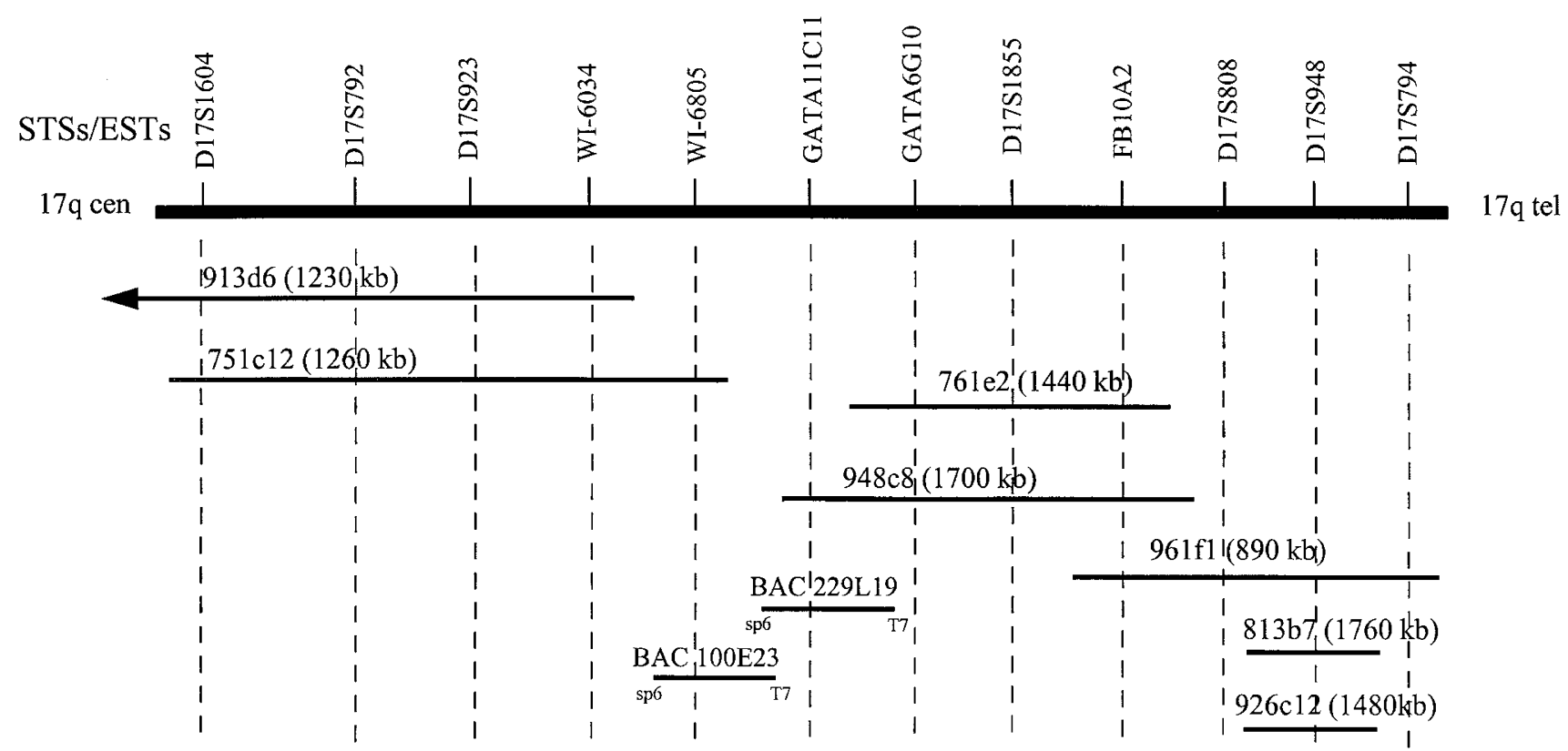

Figure 3 A diagram illustrating a YAC contig and STS marker map spanning the RP 17 critical interval. A gap in the contig is evident between markers WI-6805 and CHLC.GATA 11C 11. Investigations to cover this gap identified the two BAC clones $100 \mathrm{E} 23$ and 229L 19 
identified: BAC 100E 23 (positive for WI-6805) and BA C 229L 19 (positive for CHLC.GATA 11C 11). U sing the modified vectorette end fragment isolation method, ${ }^{8}$ an end-clone of BAC $100 E 23$ insert D NA was produced. Southern blot analysis indicated that this $500 \mathrm{bp}$ end-clone hybridised to BAC 229L 19 DNA. Similarly, an end probe derived from BAC 229L 19 was shown to hybridise to BAC 100E 23. These results therefore provide strong evidence that the two BACs overlap and, assuming that none of the YACs have an internal deletion, thereby ensure complete and overlapping clone coverage of the R P17 critical interval.

$R$ etinal fascin was considered a good candidate for the RP17 disease gene, however, the detection of multiple recombination events in both families using novel intragenic polymorphisms, provided evidence for the exclusion of this gene. Furthermore, D NA sequencing did not reveal any pathogenic mutations in the five exons of the gene.

\section{Discussion}

Since the initial mapping of the R P17 locus to a $10 \mathrm{cM}$ interval, DNA from an additional 17 family members from family RPD 8 and family RPD 19 has been collected for study. B ased on recombination analysis in the two families, R P17 has been refined to an interval of approximately $1 \mathrm{CM}$ between D17S1604 and D 17S948 on 17q22 (Figure 2).

The newly constructed R P17 Y A C/BA C contig spanning the interval lays the groundwork for the construction of a gene map for this region. A Ithough $1 \mathrm{cM}$ may be small by genetic standards, this interval may contain more than 50 separate genes (given an average gene density of five genes per $100 \mathrm{~kb}$ for the human genome). The rate-limiting step in positional cloning approaches is the identification and subsequent characterisation of genes in the critical interval. In an attempt to isolate the R P17 gene, the cD NA selection approach ${ }^{11}$ will be used to create a transcription map of the candidate region.

O ur complementary approach to genetic and physical mapping involves the testing of candidate genes suspected to lie within the critical interval. One such candidate is the retinal fascin gene which was shown to not be the adR P gene in the two 17q-linked families. The information presented here on the three novel polymorphisms in the retinal fascin gene should facilitate investigations involving this gene in other retinopathies localised to $17 q$.
A Iso under investigation are other positional candidates including the EST FB $10 A 2$ and the T-B ox 2 gene TBX $2{ }^{12,13}$ FB $10 A 2$ maps within the RP 17 YAC contig and has been found in retinal-specific CDNA libraries. The transcriptional regulator TBX 2 has been mapped to a YAC from this contig, clone $961 f 1^{12}$ and exhibits strong sequence homology, within the T-box domain, to the D rosophila optomotor blind (omb) gene. ${ }^{13} \mathrm{M}$ utations in two other T-box genes TBX 5 and TBX 3 have been shown to be responsible for Holt-Oram syndrome ${ }^{14}$ and ulnar-mammary syndrome, ${ }^{15}$ respectively.

In conclusion, these studies significantly refine the R P17 critical interval and provide the cloned genomic DNA necessary for positional cloning of the gene causative of this specific form of retinitis pigmentosa.

\section{Acknowledgements}

We gratefully acknowledge Sr Bartmann for her work in recruiting family members and the two families RPD 8 and RPD 19 for their participation in the study. We thank Nidhi Sharma for her technical assistance with the SSCP analysis. This work was funded by the Retinal Preservation Foundation of South A frica, the Foundation Fighting Blindness of U SA, the Foundation for R esearch D evelopment, the Stella and Paul Loewenstein Charitable and E ducational Trust and the $\mathrm{H}$ arry Crossley Foundation. This work was also supported by grants HG00368 (ML) and GM26091 (JB) from the National Institutes of $\mathrm{H}$ ealth.

\section{References}

$1 \mathrm{~K}$ anski JJ: Clinical Ophthalmology: A Systematic A pproach, 3rd edn. B utterworth-H eineman: B oston, 1994; ch 12.

2 D ryja TP, Li T: M olecular genetics of retinitis pigmentosa. $\mathrm{H}$ um Mol G enet 1995; 4: 1739-1743.

3 Bardien S, E benezer N, G reenberg J et al: A n eighth locus for autosomal dominant retinitis pigmentosa is linked to chromosome 17q. H um M ol G enet 1995; 4: 1459-1462.

4 X u SY, Schwartz M, Rosenberg T, G al A : A ninth locus (R P 18) for autosomal dominant retinitis pigmentosa maps in the pericentromeric region of chromosome 1 . $\mathrm{H} \mathrm{um} \mathrm{M}$ ol $\mathrm{G}$ enet 1996; 5: 1193-1197.

5 Bardien S, Ramesar R, Bhattacharya S, G reenberg J: Retinitis pigmentosa locus on $17 q$ (RP17): fine localization to $17 q 22$ and exclusion of the PDEG and TIMP2 genes. H um G enet 1997; 101: 13-17.

6 Hudson T, Stein L, G erety S et al: A n STS-based map of the human genome. Science 1995; 270: 1945-1954 with supplementary data from the Whitehead Institute/MIT Center for $\mathrm{G}$ enome R esearch, $\mathrm{H}$ uman G enetic M apping Project, D ata R elease 12 July 1997. 
7 Saishin $\mathrm{Y}$, Shimada S, Morimura $\mathrm{H}$ et al: Isolation of a CDNA encoding a photoreceptor cell-specific actin-bundling protein: retinal fascin. FEBS Letters 1997; 414: 381-386.

8 Riley J, Butler R, O gilvie D et al: A novel, rapid method for the isolation of terminal sequences from yeast artificial chromosome (YAC) clones. Nucleic A cids Res 1990; 18: 2887-2890.

9 Orita $M$, Suzuki $Y$, Sekiya T, Hayashi $K$ : Rapid and sensitive detection of point mutations and DNA polymorphisms using the polymerase chain reaction. Genomics 1989; 5: 874-879.

$10 \mathrm{D}$ ib C, Faure S, Fizames $\mathrm{C}$ et al: A comprehensive genetic map of the human genome based on 5264 microsatellites. Nature 1996; 380: 152-154.

11 L ovett M: Fishing for complements: finding genes by direct selection. Trends G enet 1994; 10: 352-357.
12 Law DJ, Gebuhr T, Garvey N, A gulnik SI, Silver LM: Identification, characterization, and localization to chromosome 17q21-22 of the human TBX 2 homolog, member of a conserved developmental gene family. Mamm Genome 1995; 6: 793-797.

13 Campbell C, Goodrich K, C asey G, B eatty B : Cloning and mapping of a human gene (TBX2) sharing a highly conserved protein motif with the Drosophila omb gene. G enomics 1995; 28: 255-260.

14 Li QY, Newbury-Ecob RA, Terret JA et al: Holt-Oram syndrome is caused by mutations in TBX 5 , a member of the Brachyury (T) gene family. Nat Genet 1997; 15: 21-29.

15 Bamshad M, Lin RC, Law DJ et al: Mutations in human TBX 3 alter limb, apocrine and genital development in ulnar-mammary syndrome. $\mathrm{N}$ at G enet 1997; 16: 311-315. 